\title{
Rapamycin Promotes the Survival and Adipogenesis of Ischemia-Challenged Adipose Derived Stem Cells by Improving Autophagy
}

\author{
Chichi Lia Lechi Ye ${ }^{b}$ Li Yang ${ }^{c} \quad$ Xiaofang Yu Yucang He $^{\mathrm{a}}$ Zhuojie Chen ${ }^{\mathrm{a}}$ \\ Liqun Lia Dan Zhang ${ }^{\mathrm{c}}$
}

\begin{abstract}
aDepartment of Plastic Surgery, The First Affiliated Hospital of Wenzhou Medical University, Nanbaixiang, Wenzhou City, Zhejiang Province, 'bepartment of Colorectal \& Anal Surgery, The First Affiliated Hospital of Wenzhou Medical University, Nanbaixiang, Wenzhou City, Zhejiang Province, 'Department of Respiratory Medicine, The First Affiliated Hospital of Wenzhou Medical University, Nanbaixiang, Wenzhou City, Zhejiang Province, PR. China
\end{abstract}

\section{Key Words}

Rapamycin • Autophagy • Adipose-derived stem cells • Ischemia • Adipogenesis

\begin{abstract}
Background/Aims: Ischemia is one of the main causes of the high rate of absorption of transplanted autologous fat. Autophagy allows cells to survive by providing energy under starvation. Rapamycin has been found to play a role in promoting autophagy. In this study, we investigated whether rapamycin participates in the survival and adipogenesis of ischemia-challenged adipose-derived stem cells (ADSCs) by regulating autophagy. Methods: Before the cells were exposed to oxygen-glucose deprivation (OGD), a simulated ischemic microenvironment, the level of autophagy was reduced or increased by lentiviral transfection with short hairpin RNA targeting microtubule-associated protein 1-light chain 3 gene (shRNALC3) or treatment with rapamycin, respectively. The level of autophagy was assessed by western blotting, transmission electron microscopythen the apoptosis ratio was determined through terminal deoxynucleotidyl transferase-mediated dUTP nick-end labeling (TUNEL) and flow cytometry. Adipogenesis was further evaluated by oil red $O$ staining and the expressions level of some specific proteins for adipocytes. Results: shRNA-LC3 and rapamycin treatment effectively decreased and improved the level of autophagy in cells with or without OGD challenge, respectively. In addition, autophagy inhibition increased the apoptosis rate and activated caspase-3 expression level in response to OGD, and these were markedly inhibited by rapamycin preconditioning. During adipogenesis, autophagy inhibition decreased not only oil droplet accumulation but also lipoprotein lipase (LPL) and peroxisome proliferatoractivated receptor gamma (PPARY) expression in cells with or without OGD challenge. However, autophagy promotion by rapamycin increased oil droplet accumulation and LPL and PPARY
\end{abstract}

Liqun Li

and Dan Zhang 


\section{Cellular Physiology Cell Physiol Biochem 2017;44:1762-1774 \\ \begin{tabular}{l|l} 
and Biochemistry Published online: December 06, 2017 & $\begin{array}{l}\text { C } 2017 \text { The Author(s). Published by S. Karger AG, Basel } \\
\text { www.karger.com/cpb }\end{array}$
\end{tabular} \\ Li et al.: Rapamycin Promotes the Adipogenesis of Adipose Derived Stem Cells}

expression. Conclusions: Rapamycin may promote the survival and adipogenesis of ischemiachallenged ADSCs by upregulating autophagy.

\section{Introduction}

Due to their abundance and ease of access, fat cells have been widely used in maxillofacial and plastic surgery for filling in defects or malformations after facial skin transplantation. However, the low survival rate of the transplanted fat cells after this procedure leads to unsatisfactory therapeutic effects. Some studies have shown that after transplantation, most of the surviving adipocytes are derived from adipose-derived stem cells (ADSCs) and are not the original transplanted adipocytes. It has been reported that transplanted fat tissues usually cannot survive for long if ADSCs are removed from these tissues in advance $[1,2]$. Therefore, more and more investigators have begun to focus on the transplantation of ADSCs and adipocytes rather than that of mature adipocytes alone [3-5]. Although the transplantation of combined ADSCs and adipocytes has shown better therapeutic effects, approximately $25 \%$ to $80 \%$ of the transplanted cells have been reported to be absorbed after transplantation [6]. Therefore, it is important to discover ways to promote the survival of transplanted ADSCs under ischemic conditions.

Autophagy is the catabolic process of cytosolic organelle and protein degradation. Under physiological conditions, the autophagy level is very low, but it can increase in response to starvation, ischemia and other stresses [7]. Autophagy has been shown to play important roles in regulating the nutrient supply and cell death during short-term starvation, ischemia and hypoxia [8]. In addition, recent studies have demonstrated that autophagy participates in regulating cellular differentiation $[9,10]$. Nevertheless, it is unclear whether autophagy participates in regulating the survival and adipogenesis of ADSCs in an ischemic microenvironment.

Rapamycin is an immunosuppressant that is widely used for inhibiting the immune rejection of transplanted organs. In addition, it is one of the most effective enhancers of autophagy and functions primarily by specifically inhibiting the mammalian target of rapamycin complex 1 [11]. Some studies have suggested that rapamycin exhibits cytoprotective effects against a variety of diseases by regulating autophagy [12, 13]. Therefore, we speculate that preconditioning with rapamycin regulates the survival of ADSCs and their differentiation into adipocytes by mediating autophagy.

In this study, we inhibited or promoted autophagy in ADSCs before challenging them with oxygen-glucose deprivation (OGD) to mimic an ischemic microenvironment in vitro. After the OGD challenge, cellular apoptosis and adipogenesis was evaluated to explore the role of autophagy in regulating the survival and adipogenesis of ADSCs in an ischemic microenvironment. Our study will be helpful for not only clarifying the role of autophagy in maintaining the survival of ADSCs in an ischemic microenvironment but also exploring the mechanism and role of autophagy in regulating the differentiation of ADSCs into adipocytes.

\section{Materials and Methods}

Experimental cells

Human ADSCs were purchased from Cyagen Biosciences Technology and cultured in ADSC complete growth medium (Cyagen, Guangzhou, Guangdong, China). When the cells had grown to $70 \%-80 \%$ confluence, they were harvested with trypsin. After centrifugation, the cells were plated on new culture dishes at approximately 6000 cells $/ \mathrm{cm}^{2}$.

Design and vector preparation of inducible lentiviral shRNAs

To generate shRNA-expressing plasmids, double-stranded oligonucleotides encoding the desired shRNA were cloned into the AgeI and EcoRI restriction sites of the pLKO-Tet-On vector (Addgene, Inc., Cambridge, 


\section{Cellular Physiology Cell Physiol Biochem 2017;44:1762-1774 \begin{tabular}{l|l} 
DOI: 10.1159/000485783 & $\begin{array}{l}\text { O 2017 The Author(s). Published by S. Karger AG, Basel } \\
\text { www.karger.com/cpb }\end{array}$ \\
\hline
\end{tabular}}

Li et al.: Rapamycin Promotes the Adipogenesis of Adipose Derived Stem Cells

MA, USA). Constructs containing an LC3-specific shRNA sequence 5'-CTGAGATCGATCAGTTCAT-3' and scrambled shRNA 5'-GCAAGCTGACCCTGAAGTTCAT-3' were designated pLKO-Tet-shRNA-LC3 and pLKO-TetshRNA-Con, respectively.

Virus production and transfection of target cell lines

Lentiviruses were generated by co-transfecting 293T cells with $1.5 \mu \mathrm{g}$ shRNA-encoding plasmids, 1 $\mu \mathrm{g}$ pPAX2 and $0.5 \mu \mathrm{g}$ pDMG2 (Addgene, Inc. Cambridge, MA) as helper plasmids using Lipofectamine 3000 reagent (Invitrogen Life Technologies) according to the manufacturer's instructions. The growth medium was changed after 8-16 h, and the lentivirus-containing supernatant was harvested after 24, 48 and $72 \mathrm{~h}$.

For transduction, the target cells were passaged to $40 \%$ confluence the following day. Lentiviralcontaining medium was added to the cells with $8 \mu \mathrm{g} / \mathrm{ml}$ polybrene (Sigma-Aldrich). After $24 \mathrm{~h}$, the viral particle-containing medium was removed and replaced with fresh medium containing $1 \mu \mathrm{g} / \mathrm{ml}$ puromycin. From days 4 to 10, the medium was replaced when necessary, and the cells were evaluated for cytotoxicity under a microscope. Finally, the cells were collected for further experiments and referred to as hADSCs shRNALC3 and hADSCs ${ }^{\text {shRNA-Con }}$.

\section{Quantification of $m R N A$ using real-time RT-PCR}

For verifying the effectiveness of shRNA-LC3 on the expression of LC3 in ADSCs, total RNA was isolated from the cells by using a GenElute Mammalian Total RNA Kit (Sigma, St. Louis, MO) according to the manufacturer's instructions. The purified RNA was then used for reverse transcription using One Step reverse transcription polymerase chain reaction (TaKaRa) and primers specific for LC3 (forward: 5'-GAGAAGCAGCTTCCTGTTCTGG-3' and reverse: 5'-GTGTCCGTTCACCAACAGGAAG-3') or GAPDH (forward: 5'-GGGTGTGAACCATGAGAAGT-3' and reverse: 5'-GTAGAGGCAGGGATGATGTT-3') as the control to verify RNA integrity. The reactions were run for 35 cycles, and the products were separated on a $2 \%$ agarose gel. Relative gene expression was calculated as follows: First, the cycle threshold (Ct) value for each target gene was determined and corrected by subtracting the $\mathrm{Ct}$ for GAPDH $(\Delta \mathrm{Ct}$ ) for each sample assayed (the $\mathrm{Ct}$ value represents the cycle when the sequence detection application began to detect an increase in the signal associated with an exponential growth of the PCR product). Second, untreated controls or initial values of the samples were chosen as reference samples, and the $\Delta \mathrm{Ct}$ for all the treated experimental samples were subtracted from the $\Delta \mathrm{Ct}$ for the control samples $(\Delta \Delta \mathrm{Ct})$. Finally, the mRNA abundance of the target gene relative to the control was calculated by the formula $2^{\wedge}(-\Delta \Delta \mathrm{C} t)$. All the assays were performed in duplicate.

\section{Effect of inhibition of autophagy on the proliferation of ADSCS}

We used the 3-(4, 5-dimethylthiazol-2-yl)-2, 5-diphenyltetrazolium bromide (MTT, Sigma-Aldrich) assay to assess the proliferation of ADSCs after inhibiting autophagy by shRNA-LC3. The infected cells were seeded in a 96-well plate at a density of 2000 cells/well. MTT in phosphate-buffered saline (PBS) was added to each well $(5 \mathrm{mg} / \mathrm{ml})$, and the cells were incubated at $37^{\circ} \mathrm{C}$ for $4 \mathrm{~h}$. The supernatant was removed, and $100 \mathrm{ml}$ of dimethyl sulfoxide was added, followed by shaking for $10 \mathrm{~min}$ to dissolve the crystals. The optical density (OD) of each well was determined at $490 \mathrm{~nm}$ with a spectrophotometer. Assays were performed in triplicate at each time point.

\section{Rapamycin pretreatment}

According to our previous study [14], $10 \mathrm{nmol} / \mathrm{L}$ of rapamycin (LC Laboratories) was added into the culture medium, and the cells were subsequently incubated for $4 \mathrm{~h}$ before they were challenged with OGD.

Western blot

Briefly, the isolated cells were harvested and rinsed twice in ice-cold PBS, and then the cells were homogenized in lysis buffer (RIPA, Beyotime Institute of Biotechnology, China) supplemented with $1 \mathrm{mM}$ Halt phosphatase inhibitor cocktail for $1 \mathrm{~h}$. The homogenate was incubated on ice for 45 min and then centrifuged at $4^{\circ} \mathrm{C}(12,000 \mathrm{rpm}$ for $5 \mathrm{~min})$. Finally, the concentration of proteins in the supernatant was determined by using the BCA Protein Assay Kit according to manufacturer's instruction. Equal amounts of total protein were separated by 15\% SDS-PAGE and transferred to PVDF membranes (Immobilon-P, Millipore). Target proteins were probed with primary antibodies against LC3 (Cell Signaling Technology), 


\section{Cellular Physiology Cell Physiol Biochem 2017;44:1762-1774 \begin{tabular}{l|l|l} 
DOI: 10.1159/000485783 & $\begin{array}{l}\text { O 2017 The Author(s). Published by S. Karger AG, Basel } \\
\text { www.karger.com/cpb }\end{array}$
\end{tabular}}

Li et al.: Rapamycin Promotes the Adipogenesis of Adipose Derived Stem Cells

p62 (Cell Signaling Technology), cleaved caspase-3 (Cell Signaling Technology), lipoprotein lipase (LPL) (Abcam), and peroxisome proliferator-activated receptor gamma (PPAR $\gamma$ ) (Cell Signaling Technology). Meanwhile, GAPDH was used as the loading control. Horseradish peroxidase-conjugated secondary antibodies were applied for $1 \mathrm{~h}$, followed by protein visualization with Pierce ECL Western Blotting Substrate and autoradiography. The blots were analyzed with Quantity One 4.6 software, and the data were normalized to GAPDH and expressed as OD integration.

\section{Simulation of an ischemic microenvironment in vivo}

An OGD method was used to simulate an ischemic microenvironment in vitro according to a previous study [14]. The cells were subjected to OGD by replacing the adipogenic medium with DMEM deprived of glucose and fetal calf serum and then placed in an anaerobic chamber $\left(5 \% \mathrm{CO}_{2} / 95 \% \mathrm{~N}_{2}\right.$; BioSpherix, $\left.\mathrm{C}-174\right)$ at $37^{\circ} \mathrm{C}$ for $8 \mathrm{~h}$, and then the cells were collected for further experiments.

\section{Analysis of autophagy ultrastructures by transmission electron microscopy}

After exposure to OGD, the cells were prefixed with $2.5 \%$ glutaraldehyde in $0.1 \mathrm{M} \mathrm{PBS}$ at $4{ }^{\circ} \mathrm{C}$ overnight, post-fixed in 1\% buffered osmium tetroxide, dehydrated in graded alcohols, embedded in Epon 812, sectioned with an ultramicrotome and stained with uranyl acetate and lead citrate. Autophagosomes and autophagolysosomes of the cells were observed with a transmission electron microscope (Philips, CM120). A total of 30 sections were prepared for electron microscopy, and the autophagic structures in each group were examined in 200 cells.

Terminal deoxynucleotidyl transferase-mediated dUTP nick-end labeling (TUNEL)

To assess apoptosis in cells in response to OGD, TUNEL staining was performed according to the manufacturer's instructions (Beyotime Institute of Biotechnology, China). The cells were washed twice with ice-cold PBS, fixed with 4\% paraformaldehyde for $30 \mathrm{~min}$, washed once with PBS and then permeabilized with $0.1 \%$ Triton $\mathrm{X}-100$ in $0.1 \%$ sodium citrate for $2 \mathrm{~min}$ on ice. Then, $50 \mu \mathrm{L}$ of TUNEL staining solution was added to the wells, and the cells were incubated at $37^{\circ} \mathrm{C}$ for $1 \mathrm{~h}$ in humidified atmosphere in the dark. Then, the apoptotic cells were detected by fluorescence microscopy (200x, excitation wavelength: $488 \mathrm{~nm}$, emission wavelength: $530 \mathrm{~nm}$ ), and the apoptotic rate was analyzed. The cells with green fluorescence were defined as apoptotic cells.

\section{Apoptosis detection by flow cytometry (FCM)}

According to the instructions for the Annexin V-FITC Apoptosis Detection Kit (BD Pharmingen ${ }^{\mathrm{TM}}$ ), cells were washed twice with cold PBS and then resuspended in $1 \times$ binding buffer at a concentration of $1 \times 10^{6} \mathrm{cells} / \mathrm{ml}$. One hundred microliters of solution was transferred to $5-\mathrm{ml}$ culture tubes, followed by the addition of $5 \mu \mathrm{l}$ of Annexin V-FITC and $5 \mu \mathrm{l}$ of propidium iodide. The resulting solution was incubated for 15 minutes at room temperature in the dark followed by the addition of $400 \mu \mathrm{l}$ of $1 \times$ binding buffer. Apoptosis rates were immediately analyzed by performing FCM (BD Biosciences, FACS Aria III).

\section{Oil red 0 staining}

Adipogenic differentiation assays were performed using Human Mesenchymal Stem Adipogenic Differentiation Medium (Cyagen Biosciences) according to the manufacturer's instructions. Adipogenic differentiation was confirmed by the formation of neutral lipid vacuoles that were stained with Oil red 0 (Sigma). The cells from different groups were washed with PBS and then fixed with $10 \%$ formaldehyde for $1 \mathrm{~h}$. Then, Oil red 0 solution in isopropanol was added into the wells, and the plates were incubated for $2 \mathrm{~h}$. After removing the Oil red 0 solution, the cells were washed three times with deionized water followed by measurement of OD at $490 \mathrm{~nm}$ with a spectrophotometer.

\section{Statistical analysis}

Independent experiments were repeated three times. SPSS 13.0 software was used for processing the data. The results are presented as the mean \pm standard deviation (mean \pm SD). Comparisons between multiple groups were performed by one-way analysis of variance. $\mathrm{P}<0.05$ was considered statistically significant. 
Results

Validating the LC3 expression knockdown efficiency of shRNA-LC3 in $h A D S C s$

To assess the knockdown of LC3 expression, the cells from different groups were cultured for $72 \mathrm{~h}$ and analyzed by RT-PCR. Compared with the blank control cells and ADSCs $s^{\text {shRNA-Con, }}$ the ADSC $s^{\text {shRNA-LC3 }}$ exhibited significantly downregulated LC3 mRNA expression (Fig. 1A). Consistently, the LC3 protein expression in the ADSC $s^{\text {shRNA-Con }}$ and ADSC$\mathrm{s}^{\text {shRNA-LC3 }}$ was similar to and significantly less than that in the blank control cells, respectively (Fig. 1B).

Effect of autophagy inhibition on proliferation

The MTT assay was used to detect the effect of autophagy inhibition on ADSC proliferation. There was no significant difference in proliferation between the ADSC $s^{\text {shRNA-Con }}$ and ADSCs ${ }^{\text {shRNA-LC3 }}$ at any time point from the first day to the seventh day after transfection (Fig. 2). These data suggest that blocking autophagy by suppressing LC3 expression does not affect ADSC proliferation.

ShRNA-LC3 and rapamycin preconditioning inhibit and improve the level of autophagy in OGD-challenged cells, respectively

LC3 is a key gene in the autophagy process. A cytosolic form of LC3 (LC3-I) is conjugated to phosphatidylethanolamine to form the LC3 phosphatidylethanolamine conjugate (LC3II), which is recruited to autophagosomal membranes. Therefore, the ratio of LC3-II to LC3-I is used as a marker for the level of autophagy [15]. In addition, the expression of p62 was determined as another indicator of autophagy [16]. OGD significantly increased the conver-
B

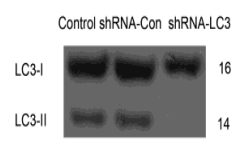

A
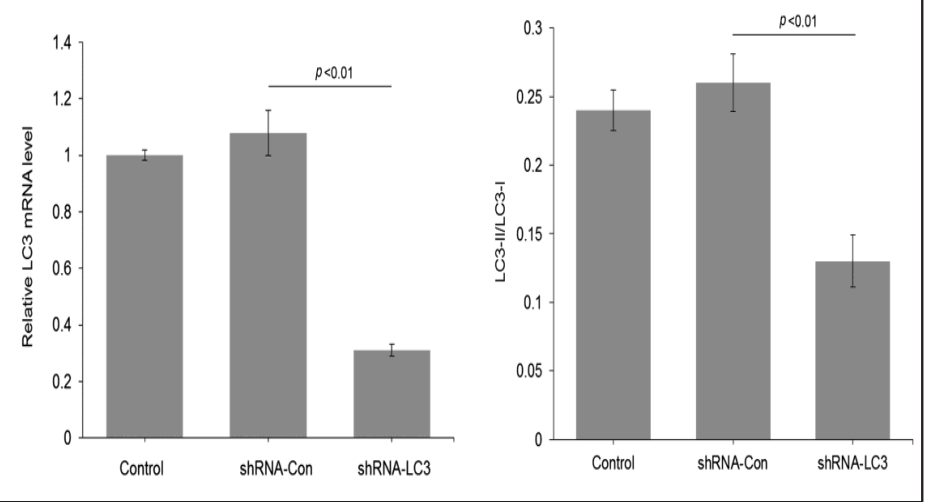

Fig. 1. Lentiviral shRNA effectively inhibited LC3 expression in hADSCs. (A) Statistical analysis of LC3 mRNA expression. (B) Representative Western blot for LC3 expression and the corresponding statistical analysis. shRNA: small hairpin RNA; LC3: microtubule-associated protein 1-light chain 3; Con: Control. The results represent the mean $\pm \mathrm{SD}(\mathrm{n}=3)$.

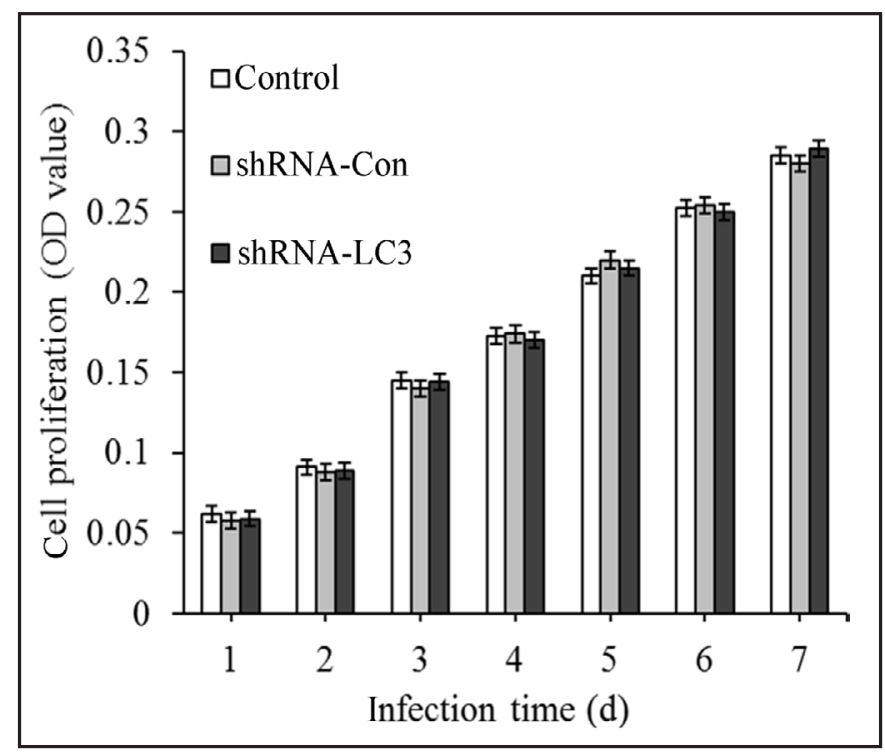

Fig. 2. Effects of lentiviral transfection with shRNA-LC3 on the proliferation of human ADSCs. The rate of hADSC proliferation gradually increased from the first day to the seventh day after the cells were transfected with shRNA-LC3. shRNA: small hairpin RNA; LC3: microtubule-associated protein 1-light chain 3; Con: Control. The results represent the mean $\pm \operatorname{SD}(n=3)$. 


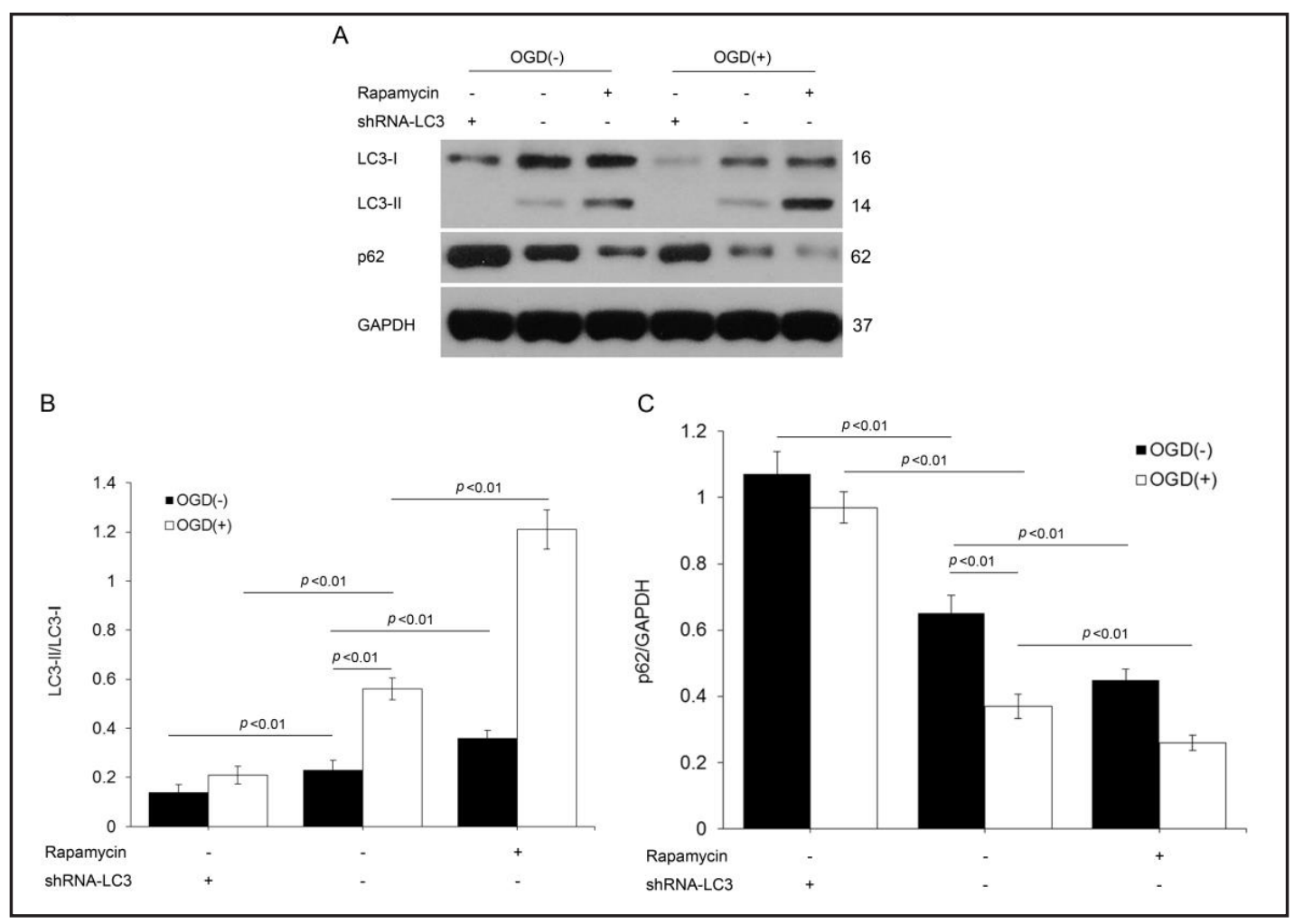

Fig. 3. The effect of lentiviral transfection with shRNA or pretreatment with rapamycin on the level of autophagy in hADSCs with or without OGD challenge. (A) Representative Western blot image showing the levels of LC3 and p62 in hADSCs challenged with OGD. (B) Statistical analysis of the ratio of LC3-II to LC3-I. (C) Statistical analysis of the expression of p62. shRNA: small hairpin RNA; LC3: microtubule-associated protein 1 -light chain 3 . The results represent the mean $\pm \mathrm{SD}(\mathrm{n}=3)$. The molecular mass is shown in $\mathrm{kDa}$.

sion of LC3-I to LC3-II and decreased p62 expression. Under both the normal and OGD conditions, shRNA-LC3 treatment effectively suppressed the autophagy level, which was reflected in the decreased conversion of LC3-I to LC3-II and the elevated p62 expression $(p<0.01)$. However, rapamycin preconditioning markedly promoted the conversion of LC3-I to LC3-II but decreased the expression of p62 in normal living and OGD-challenged cells (Fig. 3A-C). These data suggest that shRNA-LC3 and rapamycin preconditioning may effectively lower and improve the autophagy level, respectively.

ShRNA-LC3 or rapamycin preconditioning affects autophagic ultrastructures in ADSCs

In this study, we observed autophagosomes as oval structures composed of cytoplasm-like substances enclosed by a double- or multilayered membrane (Fig. 4A). Autophagolysosomes are monolayered structures, parts of which are degraded by lysosomes. Compared with the blank control cells, the OGD-treated cells exhibited more autophagosomes. Under both the normal and OGD conditions, shRNA-LC3 and rapamycin treatment significantly lowered and increased autophagosome formation, respectively ( $p<0.01$ ) (Fig. 4B).

Autophagy inhibition or promotion aggravates or alleviates OGD-induced apoptosis in ADSCs, respectively

The TUNEL assay was used to determine the apoptosis rate. In normal living cells, LC3-shRNA or rapamycin did not significantly affect the apoptosis rate. Compared with the blank control cells ( $4.95 \pm 0.96 \%)$, the OGD-treated cells exhibited an increased apoptosis rate $(13.50 \pm 1.07 \%)$. This rate further increased in $\operatorname{ADSCs}^{\mathrm{LC} 3 \text {-shRNA }}(22.3 \pm 1.04 \%)$ but was decreased $(7.36 \pm 1.13 \%)$ in the cells pretreated with rapamycin (Fig. 5A, B). 


\section{Cellular Physiology Cell Physiol Biochem 2017;44:1762-1774 \begin{tabular}{l|l} 
DOI: 10.1159/000485783 & $\begin{array}{l}\text { O 2017 The Author(s). Published by S. Karger AG, Basel } \\
\text { wwww.karger.com/cpb }\end{array}$
\end{tabular} \\ Li et al.: Rapamycin Promotes the Adipogenesis of Adipose Derived Stem Cells}

In another experiment, we further tested changes in the apoptosis rate through FCM. OGD challenge increased the apoptosis rate from $4.19 \pm 0.13 \%$ to $15.44 \pm 1.22 \%$. Inhibiting or promoting autophagy further increased or decreased the index to $23.74 \pm 1.47 \%$ or 7.78 $\pm 1.19 \%$, respectively (Fig. 5 C, D).

\section{Effect of autophagy on cleaved caspase-3 expression}

To further validate the role of rapamycin and autophagy in hADSC apoptosis in response to OGD, we assessed changes in the expression of cleaved caspase-3, an activated form of caspase-3. Under normal conditions, shRNA-LC3 or rapamycin preconditioning did not significantly affect cleaved caspase- 3 expression. Compared with the control cells, the OGDchallenged cells exhibited upregulated cleaved caspase- 3 levels, and this was significantly exacerbated by shRNA-LC3 but inhibited by rapamycin pretreatment (Fig. 6A, B).

Inhibiting or promoting autophagy suppresses or facilitates ADSC adipogenesis, respectively

Oil red 0 staining was used to assess the intracellular accumulation of oil droplets to measure ADSC adipogenesis. Compared with the blank control cells, the OGD-challenged

Fig. 4. Transmission electron microscopy images showing characteristic autophagosomes and autolysosomes in hADSCs after lentiviral transfection with shRNA or pretreatment with rapamycin with or without OGD challenge. (A) Representative transmission electron microscopy images of autophagic ultrastructures. Autophagosomes and autolysosomes are indicated by arrows and dovetail arrows, respectively. $\mathrm{N}$ represents the nucleus. (B) Quantitative analysis of the number of autophagosomes in different groups. shRNA: small hairpin RNA; LC3: microtubule-associated protein 1-light chain 3 . The results represent the mean \pm SD $(n=3)$.

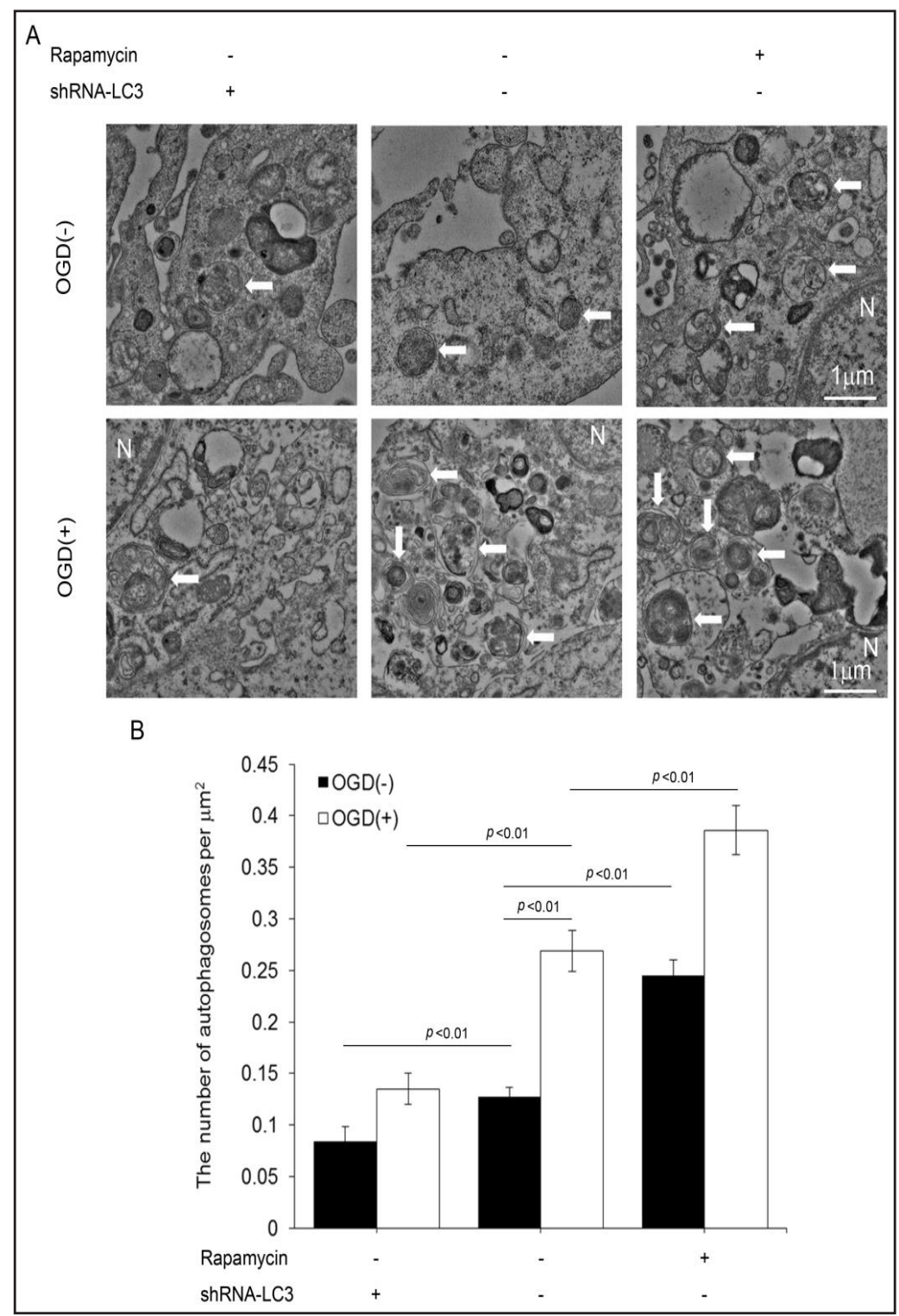




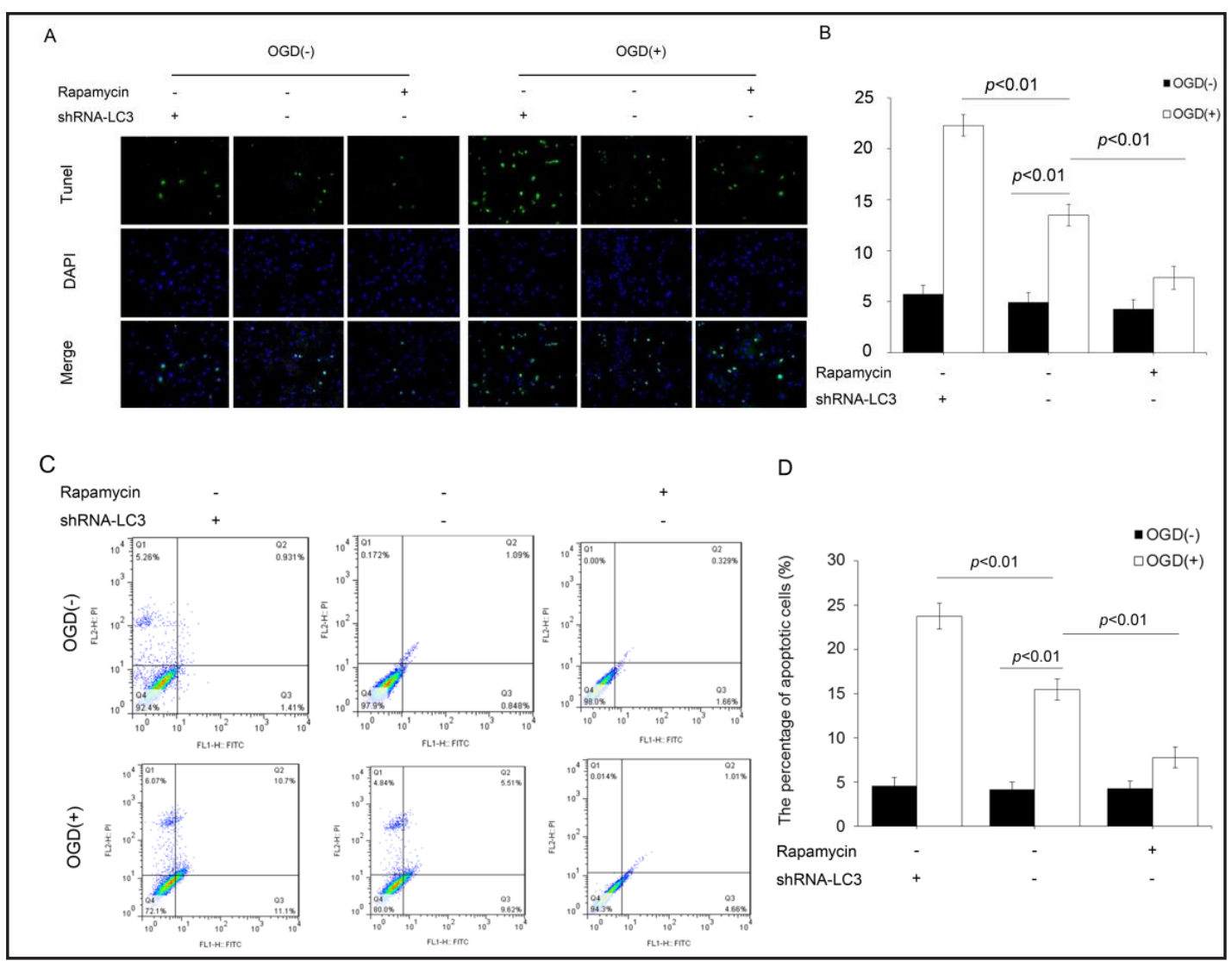

Fig. 5. The TUNEL assay was used to determine the rate of apoptosis in hADSCs after lentiviral transfection with shRNA-LC3 or pretreatment with rapamycin with or without OGD challenge. (A-B) Representative fluorescence images and corresponding statistical analysis of apoptosis detected by TUNEL. (C-D) Typical flow cytometry quadrantal diagrams and corresponding statistical data for apoptotic hADSCs. The top left, top right, and bottom right plots represent the necrotic cells and the late and early apoptotic cells, respectively. shRNA: small hairpin RNA; LC3: microtubule-associated protein 1-light chain 3. The results represent the mean \pm SD $(n=3)$.

cells exhibited markedly reduced oil droplet formation and accumulation. Under both the normal and OGD conditions, shRNA-LC3 or rapamycin pretreatment significantly suppressed or facilitated the formation of lipid droplets, respectively $(p<0.01)$ (Fig. 7A, B).

To further investigate adipogenesis in the cells, we detected changes in the expression of LPL and PPAR $\gamma$. LPL is an adipocyte-specific gene, and PPAR $\gamma$ plays an important role in adipocyte differentiation by controlling LPL expression. OGD treatment markedly lowered the expression of both LPL and PPAR $\gamma$. The inhibition or promotion of autophagy effectively decreased or increased, respectively, the expression of LPL and PPAR $\gamma$ in normal living cells. Similarly, inhibiting or promoting autophagy further suppressed or promoted the expression of both proteins under OGD (Fig. 8A-D). These findings indicate that improving the level of autophagy by rapamycin could facilitate hADSC adipogenesis under normal and simulated ischemic conditions in vitro.

\section{Discussion}

Transplanted adipocytes are under ischemic conditions before the blood supply is reestablished. An insufficient glucose and oxygen supply can be detrimental to their survival and subsequent adipogenesis [17]. Autophagy, a lysosome-dependent degradation process, 
Fig. 6. The effect of rapamycin pretreatment or shRNA-LC3 transfection on the expression of cleaved caspase-3 in hADSCs with or without OGD challenge. (A) Representative Western blot images and (B) statistical analysis of caspase- 3 and cleaved caspase-3. Representative Western blot image showing the level of cleaved caspase-3 in hADSCs. (B) Statistical analysis of cleaved caspase- 3 expression. The results represent the mean $\pm S D(n=3)$.

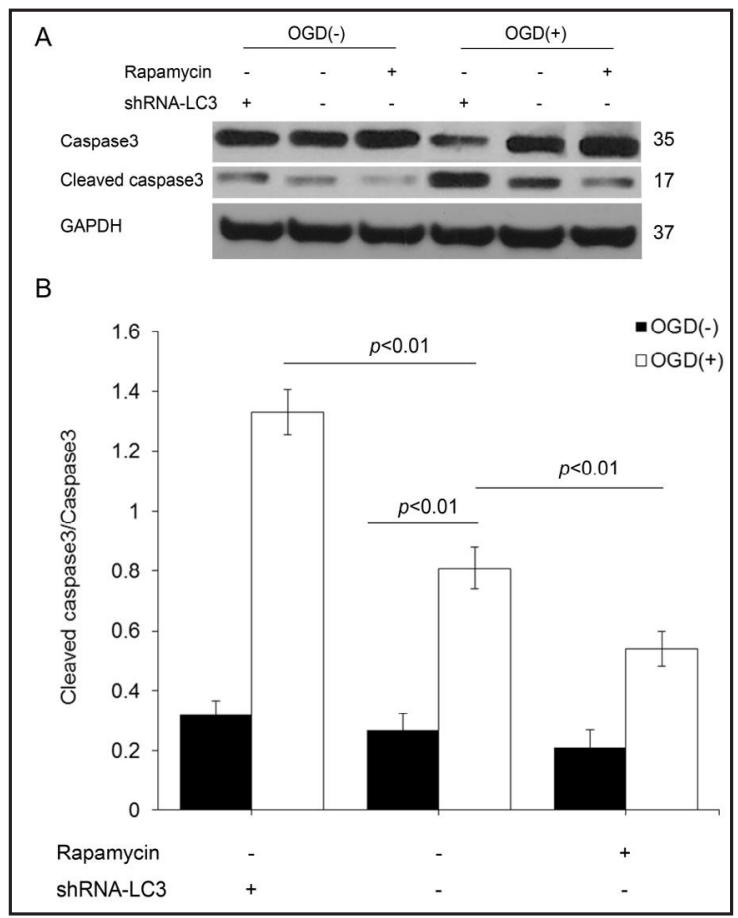

Fig. 7. The effect of lentiviral shRNA transfection or rapamycin pretreatment on the accumulation of oil droplets on day 14 in hADSCs with or without OGD challenge after adipogenic induction. (A) Representative images of Oil red 0 staining. (B) Quantitative analysis of oil droplet accumulation. shRNA: small hairpin RNA; LC3: microtubuleassociated protein 1-light chain 3 . The results represent the mean $\pm S D(n=3)$.

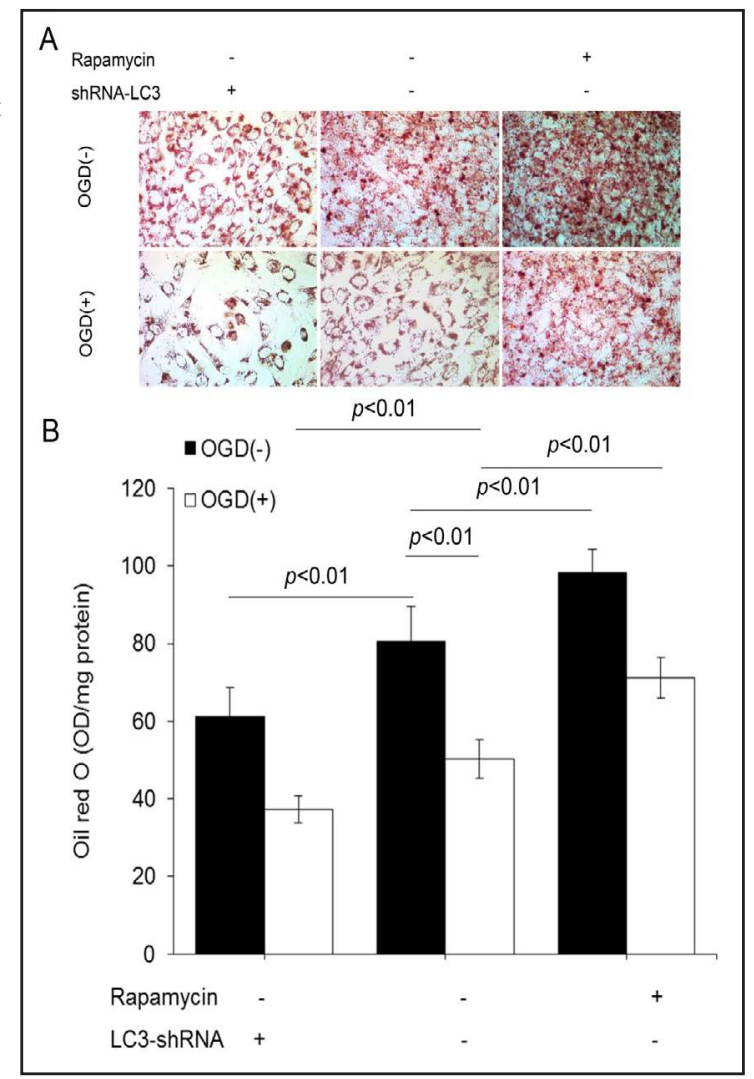

can maintain the homeostasis of the cellular environment by eliminating aging organelles and misfolded proteins [18]. Autophagy protects bone marrow-derived MSCs (BM-MSCs) from apoptosis under hypoxia [19]. Recent research has suggested that autophagy plays a pivotal role in the glucose metabolism of MSCs [20]. Based on the research described above, 


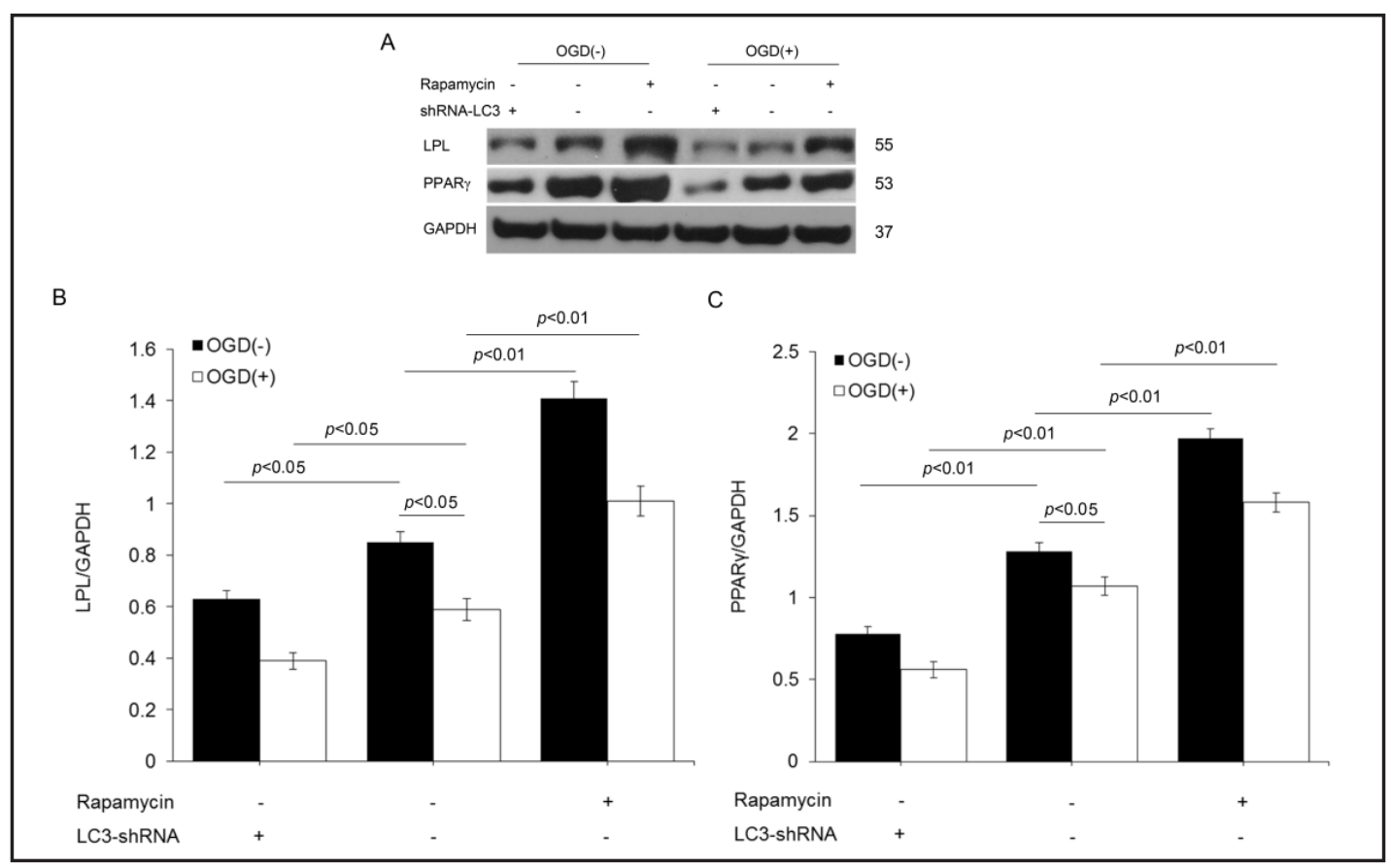

Fig. 8. Detecting the effect of lentiviral shRNA transfection or rapamycin pretreatment on the expression of LPL or PPAR $\gamma$ on day 10 in hADSCs with or without OGD challenge after adipogenic induction. (A) Representative Western blot image showing the level of LPL expression in hADSCs. (B) Statistical analysis of LPL expression. (C) Representative Western blot image showing the level of PPAR $\gamma$ expression in hADSCs. (D) Statistical analysis of PPAR $\gamma$ expression. shRNA: small hairpin RNA; LC3: microtubule-associated protein 1 -light chain 3 . The results represent the mean \pm SD $(n=3)$. The molecular mass is shown in $\mathrm{kDa}$.

we posited that mediating autophagy might substantially affect the fate of ADSCs after transplantation.

Our current study showed that autophagy inhibition increased OGD-induced apoptosis but that rapamycin preconditioning reversed this effect. To confirm that the role of rapamycin in inhibiting apoptosis depends on autophagy, we also detected the effect of rapamycin pretreatment on autophagy and found that rapamycin effectively increased the autophagy level. The role of rapamycin depends on its dose. For example, low doses of rapamycin exert negligible immunosuppressive effects, whereas high doses have significant antiinflammatory effects and even facilitate the transformation of normal cells into tumor cells $[21,22]$. In previous studies, we found that low doses of rapamycin could effectively induce autophagy and reduce death and damage in pulmonary microvascular endothelial cells in an ischemic microenvironment in vitro [14]. In this study, we used a low dose of rapamycin. In addition, autophagy inhibition through shRNA-LC3 significantly aggravated the observed OGD-induced apoptosis. Therefore, we speculated that the anti-apoptotic effect of rapamycin in response to OGD depends at least partly on the role of rapamycin in promoting autophagy. In the future, it may be feasible to apply low doses of rapamycin to improve the survival rate of transplanted ADSCs due to its dual role in preventing inflammation and promoting autophagy.

Autophagy inhibition significantly aggravated OGD-induced apoptosis, suggesting that autophagy partially mitigated the apoptosis. This finding is consistent with other research results. Hypoxic preconditioning has been found to decrease the rate of BM-MSC apoptosis after transplantation and further enhance the therapeutic potential of BM-MSCs for myocardial infarction in vivo, and these effects mainly depend on autophagy upregulation [19]. Furthermore, caspase is a known inducer of apoptosis in transplanted ADSCs under 
an ischemic microenvironment. Some researchers have suggested that autophagosomal/ lysosomal involvement selectively targets and degrades specific caspase proteins under certain types of stress [23]. In the present study, we indeed detected that the expression of activated caspase- 3 was lowered further after promoting autophagy with rapamycin under OGD conditions, which suggests that autophagy may inhibit the activation or promote the degradation of caspase-3. In addition, autophagy can selectively degrade damaged mitochondria, which will release caspase activators, such as cytochrome $c$, to avoid the activation of effector caspases. All these data reasonably explain the effect of autophagy on ADSC apoptosis under OGD conditions.

In the present study, we demonstrated that autophagy plays a positive role in the differentiation of ADSCs into mature adipocytes under both normal conditions and in response to OGD. First, we thought this was partly attributable to the inhibiting effect of autophagy on ADSC apoptosis under OGD conditions. During adipogenesis, if a sufficient number of ADSCs are preadipocytes, then ADSC crosstalk with nearby cells will produce many cytokines that regulate vital signaling pathways associated with differentiation into mature adipocytes [24-26]. However, this finding may not completely explain the effect of autophagy on ADSC adipogenesis because regulating autophagy also affects adipogenesis under normal conditions, although autophagy has no significant effects on apoptosis under these conditions. These findings indicate that autophagy may play multiple roles in ADSC adipogenesis, other than apoptosis inhibition.

Oil droplet formation is one marker of ADSC adipogenesis. The inhibition of autophagy by downregulating LC3 expression significantly blocked the accumulation of oil droplets. Previous studies have demonstrated that autophagy is critical for the formation of the unique unilocular lipid droplet structure during normal white adipogenesis [27]. In addition, inhibiting autophagy decreased the expression of LPL and PPAR $\gamma$. However, promoting autophagy with rapamycin reversed these effects. Similar findings have been obtained in other studies. Younce et al. found that blocking autophagy significantly attenuated both the accumulation of lipids and the expression of LPL [28]. Another study showed that the deletion of autophagy-related gene 5 promoted proteasome-dependent PPAR $\gamma 2$ degradation and attenuated the differentiation of 3T3-L1 preadipocytes into adipocytes [29]. Moreover, ADSCs undergo marked changes in morphology and gene expression to successfully differentiate into mature adipocytes. These morphological changes involve reorganization of the actin cytoskeleton. Recent research has suggested that the autophagy-lysosomedependent pathway may facilitate proper cytoskeletal organization through degrading the negative regulator of cytoskeletal organization [30]. All these findings suggest that autophagy is a vital process of ADSC adipogenesis under ischemic conditions.

\section{Conclusion}

In conclusion, this study found that pretreatment with a low dose of rapamycin effectively improved the level of ADSCs autophagy to alleviate OGD-induced apoptosis and ultimately promote ADSCs differentiation into mature adipocytes. These findings should be helpful for elucidating the mechanism of survival and adipogenesis of transplanted ADSCs under ischemic conditions, and they suggest that intervention with low doses of rapamycin is a feasible way to improve the therapeutic effect of fat transplantation.

\section{Acknowledgements}

Chichi Li and Lechi Ye contributed equally to this work. This work was financially supported by the National Nature Science Foundation of China (No. 81601697 and No. 81571923), the Zhejiang Provincial Natural Science Foundation of China (No. LQ15H150002), the Wenzhou Science and Technology Project (No. Y20160398). 


\section{Cellular Physiology Cell Physiol Biochem 2017;44:1762-1774 \begin{tabular}{l|l|l} 
and Biochemistry Published onlIne: December 06, 2017 & $\begin{array}{l}\text { (c) } 2017 \text { The Author(s). Published by S. Karger AG, Basel } \\
\text { www.karger.com/cpb }\end{array}$ \\
\hline
\end{tabular}}

Li et al.: Rapamycin Promotes the Adipogenesis of Adipose Derived Stem Cells

\section{Disclosure Statement}

The authors declare no Disclosure Statement.

\section{References}

$>1$ Jung DW, Kim YH, Kim T, Lee JH, Chung KJ, Lim JO, Choi JY: Improvement of Fat Transplantation: Fat Graft with Adipose-Derived Stem Cells and Oxygen-Generating Microspheres. Ann Plast Surg 2015;75:463-470.

2 Tabit CJ, Slack GC, Fan K, Wan DC, Bradley JP: Fat grafting versus adipose-derived stem cell therapy: distinguishing indications, techniques, and outcomes. Aesthetic Plast Surg 2012;36:704-713.

-3 Marwah M, Kulkarni A, Godse K, Abhyankar S, Patil S, Nadkarni N: Fat Ful'fill'ment: A Review of Autologous Fat Grafting. J Cutan Aesthet Surg 2013;6:132-138.

-4 Sterodimas A, de Faria J, Nicaretta B, Boriani F: Autologous fat transplantation versus adipose-derived stem cell-enriched lipografts: a study. Aesthet Surg J 2011;31:682-693.

5 Zhang W, Schmull S, Du M, Liu J, Lu Z, Zhu H, Xue S, Lian F: Estrogen Receptor $\alpha$ and $\beta$ in Mouse: AdiposeDerived Stem Cell Proliferation, Migration, and Brown Adipogenesis In vitro. Cell Physiol Biochem 2016;38:2285-2299.

6 Kølle SF, Fischer-Nielsen A, Mathiasen AB, Elberg JJ, Oliveri RS, Glovinski PV, Kastrup J, Kirchhoff M, Rasmussen BS, Talman ML, Thomsen C, Dickmeiss E, Drzewiecki KT: Enrichment of autologous fat grafts with ex-vivo expanded adipose tissue-derived stem cells for graft survival: a randomised placebocontrolled trial. Lancet 2013;382:1113-1120.

7 Yabu T, Imamura S, Mizusawa N, Touhata K, Yamashita M: Induction of autophagy by amino acid starvation in fish cells. Mar Biotechnol 2012;14:491-501.

8 Loos B, Genade S, Ellis B, Lochner A, Engelbrecht AM: At the core of survival: autophagy delays the onset of both apoptotic and necrotic cell death in a model of ischemic cell injury. Exp Cell Res 2011;317:1437-1453.

-9 Vessoni AT, Muotri AR, Okamoto OK: Autophagy in stem cell maintenance and differentiation. Stem Cells Dev 2012;21:513-520.

10 Song BQ, Chi Y, Li X, Du WJ, Han ZB, Tian JJ, Li JJ, Chen F, Wu HH, Han LX, Lu SH, Zheng YZ, Han ZC: Inhibition of Notch Signaling Promotes the Adipogenic Differentiation of Mesenchymal Stem Cells Through Autophagy Activation and PTEN-PI3K/AKT/mTOR Pathway. Cell Physiol Biochem 2015;36:1991-2002.

11 Kim YC, Guan KL: mTOR: a pharmacologic target for autophagy regulation. J Clin Invest 2015;125:25-32.

12 Sekiguchi A, Kanno H, Ozawa H, Yamaya S, Itoi E: Rapamycin promotes autophagy and reduces neural tissue damage and locomotor impairment after spinal cord injury in mice. J Neurotrauma 2012;29:946-956.

13 Urbanek T, Kuczmik W, Basta-Kaim A, Gabryel B: Rapamycin induces of protective autophagy in vascular endothelial cells exposed to oxygen-glucose deprivation. Brain Res 2014;1553: 1-11.

14 Zhang D, Li C, Zhou J, Song Y, Fang X, Ou J, Li J, Bai C: Autophagy protects against ischemia/reperfusioninduced lung injury through alleviating blood-air barrier damage. J Heart Lung Transplant 2015;34:746755.

15 Tanida I, Ueno T, Kominami E: LC3 conjugation system in mammalian autophagy: Int J Biochem Cell Biol 2004;36:2503-2518.

-16 Pankiv S, Clausen TH, Lamark T, Brech A, Bruun JA, Outzen H, Øvervatn A, Bjørkøy G, Johansen T: p62/ SQSTM1 binds directly to Atg8/LC3 to facilitate degradation of ubiquitinated protein aggregates by autophagy. J Biol Chem 2007;282:24131-24145.

17 Nishimura T, Hashimoto H, Nakanishi I, Furukawa M: Microvascular angiogenesis and apoptosis in the survival of free fat grafts. Laryngoscope 2000;110:1333-1338.

18 Kim I, Lemasters JJ: Mitochondrial degradation by autophagy (mitophagy) in GFP-LC3 transgenic hepatocytes during nutrient deprivation. Am J Physiol-Cell Ph 2011; 300:C308-C317.

19 Zhang Z, Yang C, Shen M, Yang M, Jin Z, Ding L, Jiang W, Yang J, Chen H, Cao F, Hu T: Autophagy mediates the beneficial effect of hypoxic preconditioning on bone marrow mesenchymal stem cells for the therapy of myocardial infarction. Stem Cell Res Ther 2017;8:89.

20 Nuschke A, Rodrigues M, Wells AW, Sylakowski K, Wells A: Mesenchymal stem cells/multipotent stromal cells (MSCs) are glycolytic and thus glucose is a limiting factor of in vitro models of MSC starvation. Stem Cell Res Ther 2016;7:179. 


\section{Cellular Physiology Cell Physiol Biochem 2017;44:1762-1774 \begin{tabular}{l|l} 
DOI: 10.1159/000485783 & $\begin{array}{l}\text { O 2017 The Author(s). Published by S. Karger AG, Basel } \\
\text { www.karger.com/cpb }\end{array}$
\end{tabular} \\ Li et al.: Rapamycin Promotes the Adipogenesis of Adipose Derived Stem Cells}

21 Heuer M, Benkö T, Cicinnati VR, Kaiser GM, Sotiropoulos GC, Baba HA, Treckmann JW, Broelsch CE, Paul A: Effect of low-dose rapamycin on tumor growth in two human hepatocellular cancer cell lines. Transplant Proc 2009;41:359-365.

-22 Phung TL, Eyiah-Mensah G, O’Donnell RK, Bieniek R, Shechter S, Walsh K, Kuperwasser C, Benjamin LE: Endothelial Akt signaling is rate-limiting for rapamycin inhibition of mouse mammary tumor progression. Cancer Res 2007;67:5070-5075.

23 Hou W, Han J, Lu C, Goldstein LA, Rabinowich H: Autophagic degradation of active caspase-8: a crosstalk mechanism between autophagy and apoptosis. Autophagy 2010;6:891-900.

$\checkmark 24$ Wozniak SE, Gee LL, Wachtel MS, Frezza EE: Adipose tissue: the new endocrine organ? A review article. Digest Dis Sci. 2009;54:1847-1856.

25 Xu FT, Li HM, Zhao CY, Liang ZJ, Huang MH, Li Q, Chen YC, Chi GY: Characterization of Chondrogenic Gene Expression and Cartilage Phenotype Differentiation in Human Breast Adipose-Derived Stem Cells Promoted by Ginsenoside Rg1 In vitro. Cell Physiol Biochem 2015;37:1890-1902.

26 Wang L, Xu L, Xu M, Liu G, Xing J, Sun C, Ding H: Obesity-Associated MiR-342-3p Promotes Adipogenesis of Mesenchymal Stem Cells by Suppressing CtBP2 and Releasing C/EBP $\alpha$ from CtBP2 Binding. Cell Physiol Biochem 2015;35:2285-2298.

27 Zhang Y, Goldman S, Baerga R,Zhao Y, Komatsu M, Jin S: Adipose-specific deletion of autophagy-related gene 7 (atg7) in mice reveals a role in adipogenesis. P Natl Acad Sci USA 2009;106:19860-19865.

28 Younce C, Kolattukudy P: MCP-1 induced protein promotes adipogenesis via oxidative stress, endoplasmic reticulum stress and autophagy. Cell Physiol Biochem 2012;30:307-320.

29 Zhang C, He Y, Okutsu M, Ong LC, Jin Y, Zheng L, Chow P, Yu S, Zhang M, Yan Z: Autophagy is involved in adipogenic differentiation by repressesing proteasome-dependent PPAR $\gamma 2$ degradation. Am J Physiol-endo M 2013;305:E530-E539.

-30 Shang Y, Wang H, Jia P, Zhao H, Liu C, Liu W, Song Z, Xu Z, Yang L, Wang Y, Li W: Autophagy regulates spermatid differentiation via degradation of PDLIM1. Autophagy 2016;12:1575-1592. 